\title{
Low-frequency ionospheric sounding with Narrow Bipolar Event lightning radio emissions: energy-reflectivity spectrum
}

\author{
A. R. Jacobson ${ }^{1}$, R. Holzworth ${ }^{1}$, and X.-M. Shao ${ }^{2}$ \\ ${ }^{1}$ Earth and Space Sciences, University of Washington, Seattle, WA, USA \\ ${ }^{2}$ ISR Division, Los Alamos National Laboratory, Los Alamos, NM, USA \\ Received: 7 February 2008 - Revised: 7 May 2008 - Accepted: 20 May 2008 - Published: 27 June 2008
}

\begin{abstract}
We analyze data on radio-reflection from the D-region of the lower ionosphere, retrieving the energyreflection coefficient in the frequency range $\sim 5-95 \mathrm{kHz}$. The data are the same as developed for a recent study of ionospheric-reflection height, and are based on recordings of powerful (multi-Gigawatt) radio emissions from a type of narrow $(\sim 10 \mu \mathrm{s})$ lightning discharge known as "Narrow Bipolar Events". The sequential appearance of first the groundwave signal, and then the ionospheric single-hop reflection signal, permits us to construct the energy-reflection ratio. We infer the energy reflection's statistical variation with solar zenith angle, angle-of-incidence, frequency, and propagation azimuth. There is also a marginally-significant response of the energy reflectivity to solar X-ray flux density. Finally, we review the relationship of our results to previous published reports.
\end{abstract}

Keywords. Ionosphere (Ionospheric irregularities; Plasma temperature and density; Instruments and techniques)

\section{Introduction and background}

The reflection of long radio waves from the lower ionosphere (D-region) has been studied since the 1940s, and from that perspective one might infer that anything worth doing has already been done. On the other hand, more recent work on lower-ionosphere disturbances associated with lightning (Cho and Rycroft, 1998: Inan et al., 1996a, b; Lev-Tov et al., 1995) and with lightning-induced energetic-particle precipitation (Bortniket al., 2006a, b; Rodger et al., 2005) motivate further improvements in radio reflectometry from the D-region. Present understanding of morphologies, time histories, and intensities of the electron-density disturbances associated with lightning and precipitation is impeded by in-

Correspondence to: A. R. Jacobson

(abramj@u.washington.edu) sufficiently fast and quantitative profiling of the disturbed Dregion electron density.

Systematic radio-reflection observations of the D-region were pioneered (Bracewell et al., 1951) in the United Kingdom after World War II. During the 1960s there appeared both excellent summaries of two decades of observations, and sophisticated, full-wave numerical models of the radio propagation (e.g. Deeks, 1966; Johler and Harper, 1962; Piggott et al., 1965; Pitteway, 1965; Thomas and Harrison, 1970; Wait and Spies, 1964). Whereas medium-frequency (MF, $300 \mathrm{kHz}-3 \mathrm{MHz}$ ) and, especially, high-frequency (HF, 3$30 \mathrm{MHz}$ ) ionosondes already existed and provided profiles of the E-region and F-region underside, there was no equivalent pulse-reflectometry tool for the lower ionosphere at verylow-frequency (VLF, 3-30 kHz) and low-frequency (LF, 30$300 \mathrm{kHz}$ ). Three factors prevent a D-region ionosonde: First, the low plasma frequency at D-layer heights necessitates use of LF or VLF techniques. For these frequencies, suitable radio-sounder antenna dimensions (on the order of the halfwavelength, or $\lambda / 2=5 \mathrm{~km}$ for $f=30 \mathrm{kHz}$ ) cannot be realized in practice, so that one must make do with inefficient, lowgain antennas. Second, the D-layer's high electron-neutral collision rate causes high signal loss in D-layer radio sounding, further worsening the signal-to-noise problem already implicit in the low-gain antennas. Together, in practice, these two factors prevented the ionosonde approach from being extended downward in altitude below $95 \mathrm{~km}$. Third, partial reflections are more important for the VLF (and to some extent LF) spectral range than in the higher frequencies used for conventional ionosondes.

Thus the early VLF/LF radio-sounding technique was forcedly based on narrowband bistatic carrier-phase and amplitude measurements. The narrowband radio-link approach is well typefied by the $16-\mathrm{kHz}$ observations (Bracewell et al., 1951) on the Rugby-to-Cambridge path, which were later summarized and compared to results of a full-wave model (Piggott et al., 1965). The narrowband technique is unable to discriminate partial reflections from "full" reflections.

Published by Copernicus Publications on behalf of the European Geosciences Union. 


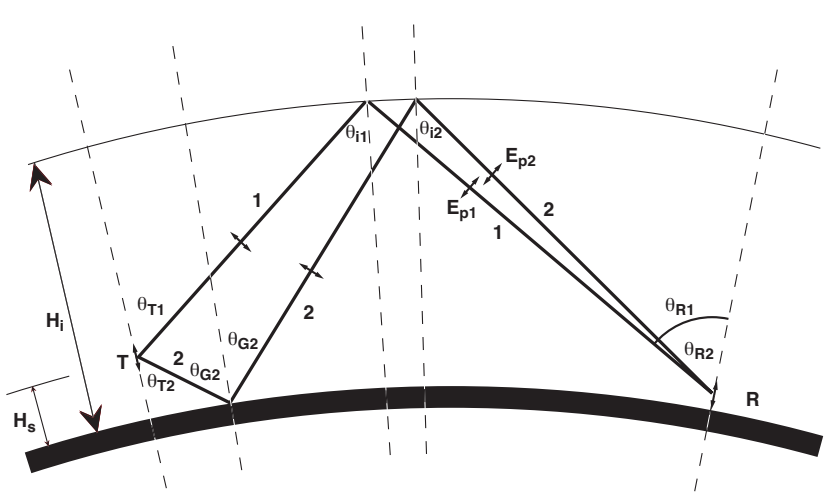

Fig. 1. Schematic of the ionospheric-reflection model. A "transmitter" $T$ (in this case an intracloud lightning discharge at height $H_{S}$ ) radiates both a ground wave (not shown) and two ionospheric echoes. The first of these echoes departs $T$ along an upward take-off angle directly to the ionosphere at height $H_{i}$. The second departs $T$ along a downward takeoff angle to the ground, where it is reflected toward the ionosphere. A ground-based receiver $R$ records all three signals, including the two echoes shown here, plus the ground wave (not shown).

In a more recent and highly successful development, narrowband carrier-phase and amplitude measurements have been applied to long-range (up to $\sim 10^{4} \mathrm{~km}$ ) propagation of signals from powerful VLF beacons (McRae and Thomson, 2000; Thomson and Clilverd, 2001: Thomson and Rodger, 2005; Thomson et al., 2004, 2007). Thomson's approach has been to compare careful recordings of phase and amplitude at long range to predictions of a numerical model of VLF propagation in the Earth-ionosphere waveguide developed under the auspices of the United States Navy (Pappert and Ferguson, 1986). In Thomson's approach, data and model resuls are compared to fit the two unknown parameters in the standard exponential model (Wait and Spies, 1964) for electron density: " $\beta$ " is the logarithmic gradient (in units of $\mathrm{km}^{-1}$ ), and $H^{\prime}$ is a reference height (in units of km.) Thomson's approach has provided accurate and stable determinations of $\beta$ and $H^{\prime}$ over long distances with pure-daytime conditions over the full path (McRae and Thomson, 2000; Thomson, 1993). The approach has more recently been successfully applied to determinations of the transient D-region perturbations of $\beta$ and $H^{\prime}$ in the sunlit hemisphere from solar flares (Thomson and Clilverd, 2001; Thomson and Rodger, 2005; Thomson et al., 2004).

An alternative approach to studying the D-region radio reflector, using wideband VLF emissions from lightning as the transmitter, rather than a narrowband artificial beacon, was developed in the late 1990s (Cummer et al., 1998). Cummer's approach exploited the immense peak radiated VLF power (multi-GW) of lightning strokes, which elegantly bypassed the obstacles to an engineered D-region pulsed sounder. This wideband VLF approach was later applied to the study of ionospheric disturbances associated with light- ning itself (Cheng and Cummer, 2005) and with particle precipitation (Cheng et al., 2006). The heart of the technique developed by Cummer is to compare the recorded against the modeled VLF spectral shape, using a form of the same numerical waveguide-propagation model as used by Thomson (Pappert and Ferguson, 1986). The sharpness parameter $\beta$ tends to control the degree of spectral interference, while the height parameter $H^{\prime}$ tends to control the frequency spacing of the modulation (Cummer et al., 1998).

In addition to Cummer's approach, some additional insight into VLF propagation in the Earth-ionosphere waveguide was provided by interpretation of long-range $(>1000 \mathrm{~km})$ sferic recordings (Grandt, 1992) in terms of a model based on dominance by the transverse-magnetic (TM) first mode. The model predicts the spectral dispersion via a group timedelay dispersion. (A "sferic" is the VLF/LF signal radiated from a lightning discharge.)

Our present approach is basically an evolution of Cummer's, in that we rely on the powerful sferic emissions rather than an engineered transmitter. We use short $(<800 \mathrm{~km})$ paths, and lightning of a special type having a sufficiently narrow pulse to permit temporal separation, in the recorded signal, between the signal components from various propagation paths. Thus our model paradigm will not be based on complicated multi-mode waveguide propagation, but rather, on the single-hop ionospheric reflection process, in isolation. We hope that, eventually, this simplification will help to illuminate the reflection process via comparison with full-wave models (e.g. an adaptation of the approach used by Pitteway, 1965) of the single reflection.

The remainder of this article will describe our technical approach (Sect. 2), present our statistical results (Sects. 3$5)$, and discuss these results in the context of prior work (Sect. 6).

\section{Technical approach}

\subsection{Basic model}

In this paper we build upon recently published results (Jacobson et al., 2007), (“J7"), on improved retrieval of ionospheric reflection height from the delayed echoes seen in recordings of VLF/LF lightning sferics of Narrow Bipolar Event (NBE) discharges (Le Vine, 1980; Smith et al., 2004; Smith et al., 1999; Willett et al., 1989). Using exactly the same reflection model as in J7, we now retrieve the magnitude (though not the phase) of the VLF/LF reflection spectrum and that magnitude's variation with frequency and with three controllable factors (angle-of-incidence, solar zenith angle, and propagation azimuth).

The reflection model is shown in Fig. 1. The NBE discharge provides a transmitter (" $T$ "), assumed to be a short dipole radiator aligned with the local vertical and elevated at height $H_{S}$ in a thundercloud. The radiation from $T$ is 
assumed to be that of a simple short dipole, with equal radiation below and above the dipole's own equatorial plane. Radiation below the plane is assumed to reflect perfectly from the ground and thence to propagate to the ionosphere (path 2) (at height $H_{i}$ ), while radiation above the dipole's equatorial plane can propagate directly to the ionosphere (path 1). The receiver (" $R$ ") is close to the ground and receives signals from both paths 1 and 2, as well as the direct, line-of-sight signal ("ground wave"). The angles of incidence at transmitter, ground, ionosphere, and receiver are shown by the symbol $\theta$ with various subscripts.

The ground wave arrives at the receiver first, and due to the narrow $(<20-\mu \mathrm{s})$ duration of the pulse, does not overlap in time with the delayed echoes from paths 1 and 2. This is crucial- we are able to measure the ground wave, and then use that as "ground truth" for converting the recorded echoes into an energy-reflection spectrum. This differs from previous uses of broadband lightning emissions for ionosphericreflection studies (Cheng and Cummer, 2005; Cheng et al., 2006; Cummer, 1997; Cummer et al., 1998), in which the ground wave and ionospheric echoes were overlapped in time. If one does not separately observe the ground-truth waveform, it is not possible to retrieve a reflection coefficient from a single sferic.

We wish to retrieve from our data the energy-reflection coefficient for the ionospheric underside, as a function of frequency $f$, angle-of-incidence $\theta$, solar zenith angle $Z$, and $T$ to- $R$ propagation azimuth (henceforth, "azimuth"). Toward this end, we make the following approximations:

1. We assume that the ground serves as a perfect conductor, and that the ground reflection dissipates no energy. We do not invoke this assumption for the ground wave, only for the second reflection in the pair of reflections. The dominant spectral attenuation in the reflection problem is due to the D-region, so this approximation is serviceable in our case.

2. We assume that the emission from $T$ is that of a vertical dipole, with radiated electric field in the plane of incidence, normal to the radial vector, and varying in magnitude as $\sin \left(\theta_{T}\right)$. Any tilts of the NBE dipole away from the local vertical would cause random errors in our final reflectivity retrieval.

3. We treat the ionospheric reflection as occurring from an "ionospheric height", whose retrieval is described in $\mathbf{J 7}$ and references therein. This height is fitted separately for each receiver's signal in each NBE lightning discharge, in a manner that phenomenologically allows for variation of radio penetration height (into the ionosphere) with range (and hence $\theta$ ), with $Z$, and with azimuth. (Our retrieved height corresponds to the "virtual height" in Eq. (3) of Piggott et al., 1965) For the purpose of stratifying the energy-reflection coefficient versus an- gle of incidence, we use an average angle of incidence, averaged over the two echo paths:

$\theta_{I}=\left(\theta_{i 1}+\theta_{i 2}\right) / 2$

4. Since the receiver is a vertical dipole near the ground, capable of sensing only a vertical electric field, we ignore that portion of the ionospherically reflected field that is normal to the plane of incidence, because that portion will not contribute to the vertical electric field at the ground. Thus the reflection coefficient we obtain is for "p-polarization", that is, with both the incident and reflected electric fields lying in the plane of incidence.

5. We sum the energy in both ionospheric echoes (paths 1 and 2) and take its ratio to the energy in the ground wave, to get the energy-reflection coefficient. This requires that we correct for modeled losses in the ground wave caused by resistive ground currents. The ground wave's dissipative attenuation is estimated by assuming a ground conductivity $\sigma_{e}=5 \times 10^{-3}$ Siemens $\mathrm{m}^{-1}$ over land and $\sigma_{e}=$ infinity over salt water, dividing the $T$ to- $R$ ground wave path into sea and land portions, and calculating the dissipative attenuation over only the land portion of that path. This is done separately for each path according to its locus on a digital map. We use a simplified formula for attenuation (see Eq. (3.13) in vol. 2, chapter 3, "Longwave Sferics Propagation within the Atmospheric Waveguide", in Volland, 1995) which suffices for estimating loss of signal power. The choice of land conductivity $\left(\sigma_{e}=5 \times 10^{-3}\right.$ Siemens $\left.\mathrm{m}^{-1}\right)$ in Volland's formula does not critically affect our results, due to the short ranges and low frequencies used here.

6. In addition to dissipative attenuation, the energy flux densities in all three signal components (ground wave, plus echoes 1 and 2) suffer dilution from spherical spreading, with electric field varying as $1 / s$, and energy flux density varying as $1 / s^{2}$, where $s$ is the path distance. We correct the ground wave energy using $s=$ the great-circle pathlength, and the sum of the echoes' energy using $s=$ the mean of the lengths of path 1 and path 2 .

7. A short dipole has an energy-flux-density lobe varying as $\sin ^{2}(\theta)$, where $\theta$ is the angle from the dipole axis (the vertical, in this case) and the radial vector to the field point. Thus the received groundwave is (approximately) at the maximum of the lobes of both the $\mathrm{T}$ and $R$ dipoles (Fig. 1.) The energy in the first and second recorded echoes, on the other hand, has been disfavored by a factor of $\sin ^{2}\left(\theta_{T 1,2}\right)$ at emission and by a factor of $\sin ^{2}\left(\theta_{R 1,2}\right)$ at reception. To correct the estimated energy in the sum of the received two echoes, we multiply the 


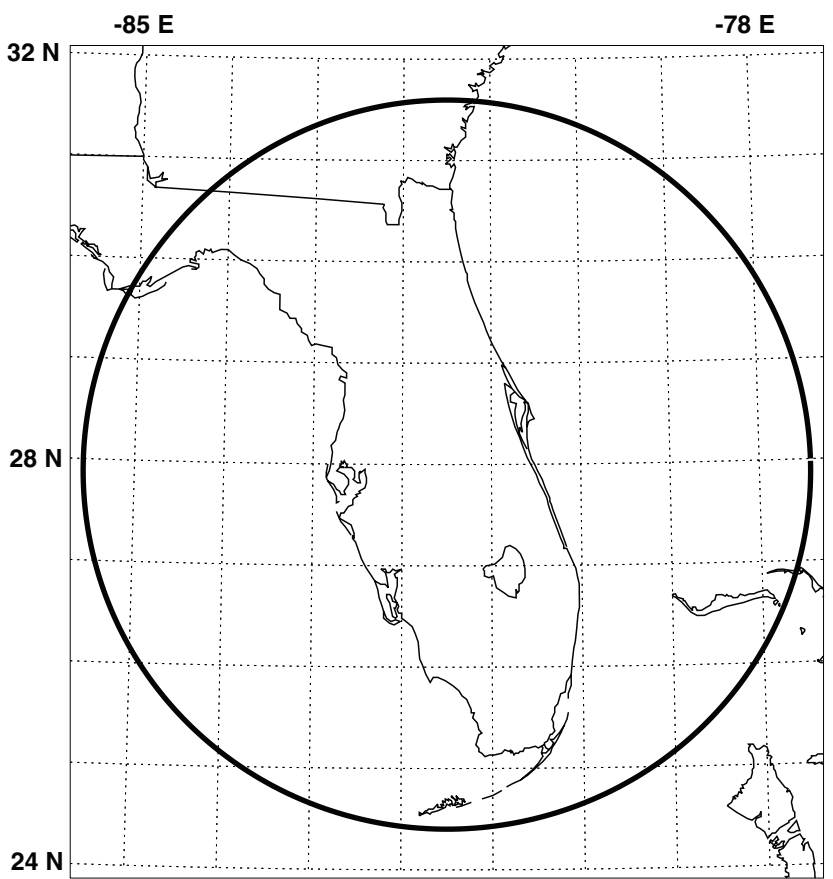

Fig. 2. Florida study area for our data. The circle has radius $400 \mathrm{~km}$ and is centered on $28 \mathrm{deg} \mathrm{N},-81.5 \mathrm{deg}$ E. Only lightning from within the circle was accepted for analysis.

received summed echo energy by a lobe-compensating factor averaged over the two paths:

$$
L=2 /\left[\sin ^{2}\left(\theta_{T 1}\right) \sin ^{2}\left(\theta_{R 1}\right)+\sin ^{2}\left(\theta_{T 2}\right) \sin ^{2}\left(\theta_{R 2}\right)\right]
$$

We reiterate that in what follows, we will use "reflectivity" to mean "energy" reflectivity of the p-polarization. This study can provide no information about either s-polarization reflection, or polarization-mixing by the reflection process.

\subsection{Data campaign}

Data are from the Florida subarray of the Los Alamos Sferic Array (Jacobson and Heavner, 2005; Jacobson et al., 2007a, b; Smith et al., 2004, 2002; Suszcynsky and Heavner, 2003) during the period 2000-2002. Figure 2 shows the area covered by this study. The Florida array in 2000-2002 was effectively centered on $28 \mathrm{deg} \mathrm{N},-81.5 \mathrm{deg}$ E. The circle in Fig. 2 is centered on that point and has radius $400 \mathrm{~km}$. In this study we use data from lightning whose locations are within that circle and from any LASA station whose distance from the lightning is $>200 \mathrm{~km}$ and $<800 \mathrm{~km}$. The data and their selection criteria are fully described in $\mathrm{J} 7$. As in the $\mathrm{J} 7$ study, we use a total of $>65000$ recorded NBEs. The 3-year campaign started at the maximum of Solar Cycle 23 (April 2000). Cyle 23's maximum was followed by two years of continued high solar flux; thus, the radio-reflection results here are thus obtained during solar-maximum-like conditions.
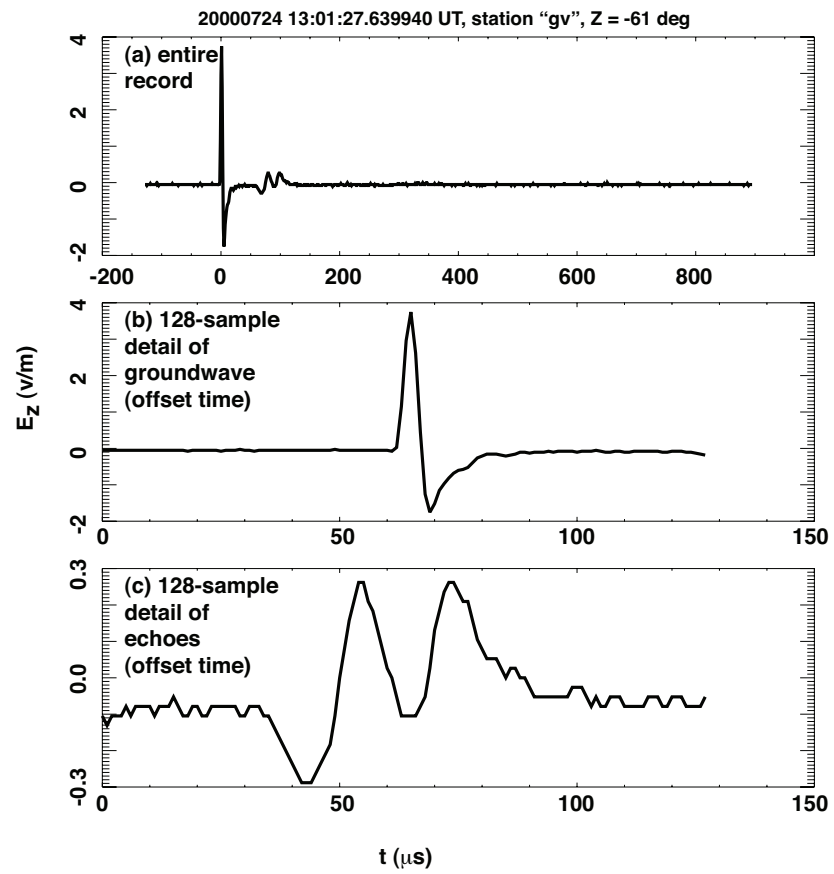

Fig. 3. Example of time-domain data from the Gainesville station, taken with solar zenith angle at ground $Z=-61 \mathrm{deg}$. (a) entire record (1000 samples at 1 sample per $\mu \mathrm{s})$, (b) 128-sample centered on the ground wave, (c) 128 samples centered on the two ionospheric echoes.

In Florida the magnetic declination is small $(-4 \mathrm{deg}$ in January 2002), and thus the geographical and geomagnetic meridians are effectively identical within the precision of this study. The local magnetic dip angle is about $58 \mathrm{deg}$.

\subsection{Data example}

Figure 3 shows time-domain data from a typical NBE recording, at the Gainesville station "gv". The solar zenith angle (at the ground) was -61 degrees, the minus sign meaning that the time was before solar-local noon, i.e. AM. Figure 3a shows 1 millisec (=1000 samples) of the record, with the trigger on the ground wave and the delayed echoes about 70 $100 \mu$ s later. Figure $3 \mathrm{~b}$ shows the 128 samples (with an offset zero) centered on the received groundwave, and Fig. 3c shows the 128 samples centered on the delayed echoes. The two windows ( $b$ and c) are time-offset to center the pulse energy optimally. This can differ from event to event. There is a small but visible background of noise. We estimate the noise using the first 120 samples in the record, that is, the pre-trigger samples preceding the ground pulse's rise (see Fig. 3a).

Taking the ratio of the echo to ground-wave spectra will give the energy-reflection coefficient as a function of frequency. Figure 4 shows frequency-resolved results for this data example. Prior to performing a Fourier transform, we 
impose a Hamming window centered on the time-domain data being analyzed. The frequency resolution is $\sim 7.8 \mathrm{kHz}$. The solid curve in Fig. 3a is the logarithm (base 10) of the power spectrum of the echoes minus the logarithm (base 10) of power spectrum of the ground wave. We have corrected the spectra in this individual plot for $1 / s^{2}$, but not for either ground-wave dissipation or dipole-lobe effects (see Eq. 2). The solid curve in Fig. $4 \mathrm{~b}$ is the logarithm of the signal-tonoise ratio (SNR), based on the recorded noise prior to the ground wave's rise.

Note that both solid curves in Fig. 4 are amplitudemodulated, with a frequency periodicity of about $50 \mathrm{kHz}$. This is the inverse of the relative delay between the two echoes (Fig. 3b), $\tau \sim 20 \mu \mathrm{s}$. The modulation of the power spectrum is an artifact of the second echo's being an approximate replica of the first echo, but with time delay $\tau$. If the second pulse were a perfect replica (which it usually is not), then we would expect the sum of the two echo pulses to have a power spectrum proportional to the power spectrum of a single pulse, with a proportionality factor

$M(\omega)=2[1+\cos (\omega \tau)]$

where $\omega=2 \pi f$. The dashed curve in Fig. $4 \mathrm{~b}$ shows this Mfactor. In order to smooth-out this modulation, we fit the energy-reflection ratio to a quadratic, shown as the dashed curve in Fig. 4a. All statistical results on energy-reflection spectra in the rest of this article will use the individual quadratic fit to the energy-reflection spectrum of each NBE recording. The interference (Eq. 3) modulation is of no interest to our study of the ionospheric reflection, and is only an artifact of our receiving two similar pulses in the spectralanalysis window, the second pulse delayed relative to the first. Examination of hundreds of case examples, analogous to that in Figs. 3 and 4, indicates that there is no "physical" spectral dependence of of the $\log _{10}$ (reflectivity) more complicated than that which can be captured by a quadratic. This approximation in effect reduces the twelve independent frequencies to just three frequency parameters (of a quadratic fit). This reduction in degrees-of-freedom is a disadvantage of our technique relative to the technique of Cummer et al. The latter retains more information about spectral response.

\section{Frequency-averaged statistical results}

\subsection{Statistical data}

We perform the spectral analysis (see Fig. 4) on each of the $N>65000$ NBE recordings. For each recording's energyreflection spectrum, we fit a quadratic to minimize the spurious modulation (see Eq. 3). The fit is applied to the frequency range $0<f<100 \mathrm{kHz}$.

We obtain in this manner an $N \times K$ matrix of spectral energy-reflection coefficients, where $K=12$ and the frequency grid is $[1,2, \ldots, 12] \times 7.8 \mathrm{kHz}$. (The sampling rate is
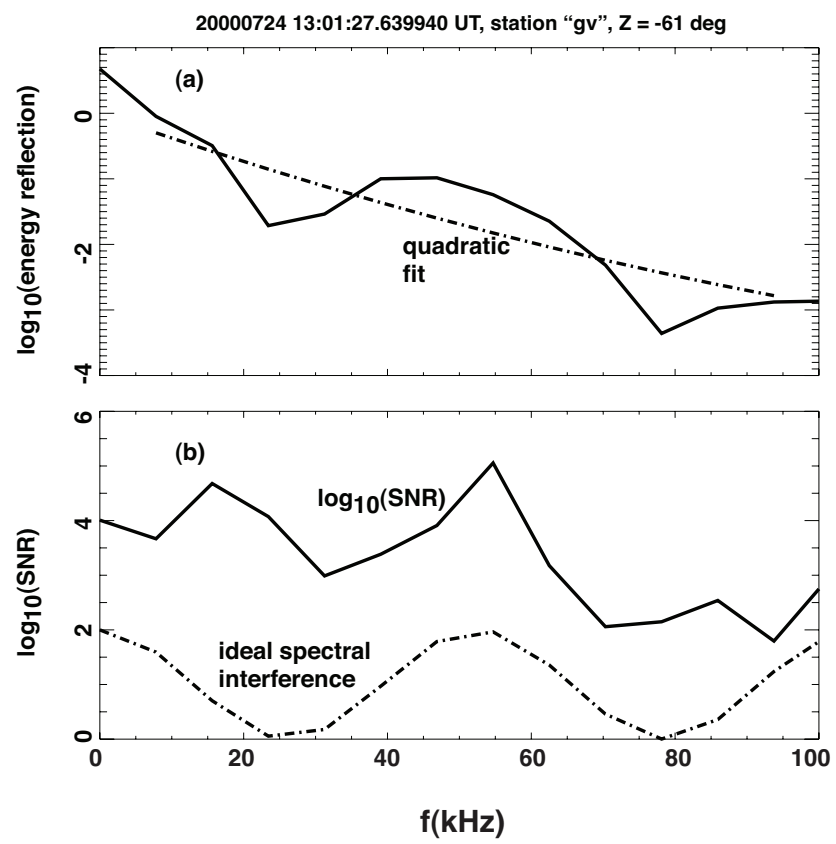

Fig. 4. Frequency-domain analysis of the data of Fig. 3. (a) $\log _{10}$ (energy reflectivity), uncorrected for dipole lobe shapes, with quadratic fit (dashed curve), (b) $\log _{10}$ (echo signal-to-noise ratio), with ideal spectral-interference model (dashed curve).

1 megasample/s, so we could in principle extend the frequencies up to the Nyquist frequency $=500 \mathrm{kHz}$, but the signalto-noise ratio is too low, above $100 \mathrm{kHz}$, for those highest frequencies to be useful, at least during daytime observations.) In addition we store the spectrum of signal-to-noise ratio (SNR), so there are two $N \times K$ data matrices serving as inputs to the statistical analyses to follow.

We use a digital map program to get the land fraction of each of the $N>65000$ ground-wave propagation paths. The energy-reflection matrix is then fully corrected for three effects:

1. The lossless energy-flux-density scaling as $1 / s^{2}$, where $s$ is the propagation pathlength;

2. the dissipative ground-wave attenuation as estimated by Volland's approximation, applied to only the land portion of each propagation path; and

3. the biases against the echo-energy estimates caused by both transmitter and receiver dipole lobes (see Eq. 2).

\subsection{Frequency bands}

In order to survey the behavior of the energy-reflection spectrum with the most robust statistics, we first divide the 0$100 \mathrm{kHz}$ frequency range into two frequency bands, lowband $(5-50 \mathrm{kHz})$ and high-band $(50-100 \mathrm{kHz})$. Each band includes six discrete multiples of $7.8 \mathrm{kHz}$ : The low band 


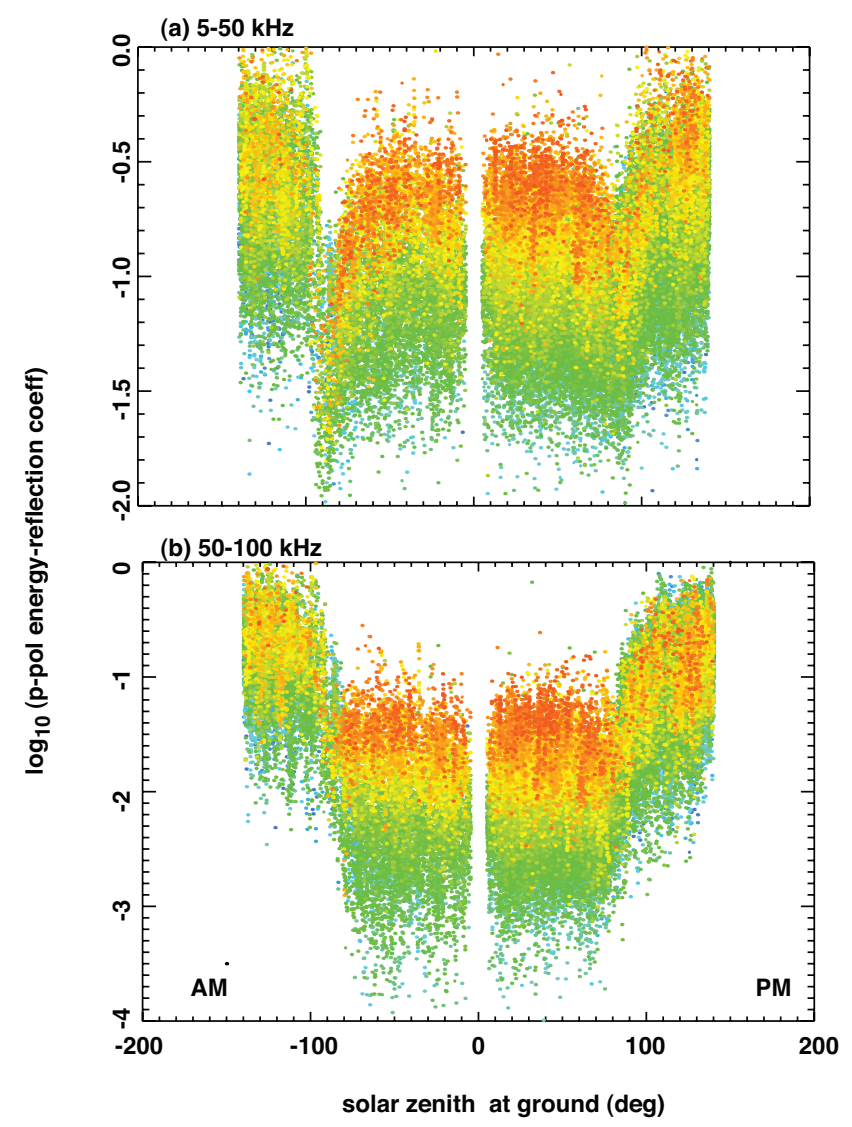

Fig. 5. $\log _{10}$ (energy reflectivity) vs. solar zenith angle. The ionospheric angle of incidence $\theta_{i}$, averaged over both echoes, is coded in color from $50 \mathrm{deg}$ (blue) to $74 \mathrm{deg}$ (red).

contains $(1,2,3,4,5,6) \times 7.8 \mathrm{kHz}$, and the high band contains $(7,8,9,10,11,12) \times 7.8 \mathrm{kHz}$.

Within each band, we average the fully-corrected quadratic fit to the reflection spectrum both over $n=1,2, \ldots$, $N$ recordings and over the six frequencies within the band. The average in each band excludes any recording/frequency entry for which the $\mathrm{SNR}<10$. In this manner we minimize the effect of noise being mistaken as echo signal, which would bias the energy reflection artificially high.

\subsection{Band-averaged results}

Figure 5 shows $\log _{10}$ (energy-reflection spectrum) versus signed solar zenith angle (AM: $<0$; PM: $>0$ ) for (a) low band and (b) high band. (Note the differing vertical scales.) There are more data during PM than during AM, due to the climatological preference of Florida-area thunderstorms for afternoon and evening.

The ionospheric angle of incidence $\theta_{i}$, averaged over both echoes, is coded in color from $50 \mathrm{deg}$ (blue) to $74 \mathrm{deg}$ (red). During daytime (say, $|Z|<80 \mathrm{deg}$ ) there is a clear progression of reflectivity upward with increasing $\theta_{i}$, while this

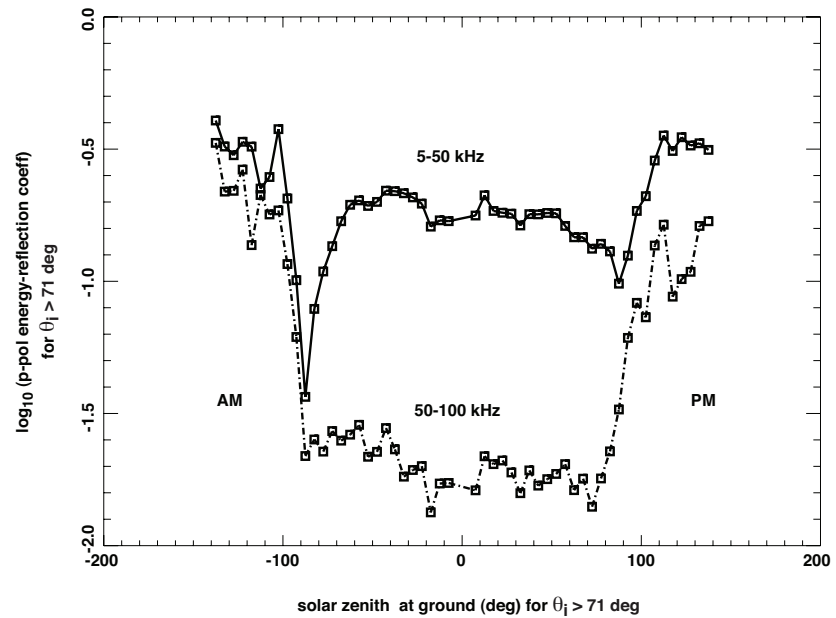

Fig. 6. Median $\log _{10}$ (energy reflectivity) vs. solar zenith angle in the most grazing angles of incidence, $71<\theta_{i}<74 \mathrm{deg}$ (the red points in Fig. 5). The signed-zenith range from -140 to $+140 \mathrm{deg}$ is divided into 56 bins, each 5-deg-wide. In zenith bins having $>100$ data points, we take the median of the energy-reflection coefficient. This median is shown for low band (solid curve) and high band (dashed curve.) The negative values of $Z$ are for rising sun (AM), the positive for falling sun (PM).

is less dramatic during night conditions (say, $|Z|>110 \mathrm{deg}$ ). The dependence on $Z$ is more visibly dramatic, with grossly higher reflectivity at night than during the day, and with sharp transitions in reflectivity at both dawn and dusk. Clearly $Z$ and $\theta_{i}$ jointly control much of the variability in the energy reflectivity.

We wish to find regressions for the $Z$ and $\theta_{i}$ dependencies of the energy reflectivity. We start by treating only the highest angles of incidence (i.e. most grazing incidence): $71<\theta_{i}<74 \mathrm{deg}$ (the red points in Fig. 5). For this grazingincidence data, we divide the signed-zenith range from -140 to +140 deg into 56 bins, each 5-deg-wide. In zenith bins having $>100$ data points, we take the median of the energyreflection coefficient. This median is shown in Fig. 6 for low band (solid curve) and high band (dashed curve.) The squares are centered on the bin centers. We note:

1. During daytime, the low- and high-band reflectivities differ by roughly an order of magnitude.

2. During nighttime, the low- and high-band reflectivities differ less, and are almost the same during pre-dawn.

3. The sunrise transition is essentially simultaneous for both the low and high bands. The transition consists of a sharp reduction in the reflectivity as the sun rises through $Z \sim-100 \mathrm{deg}$, illuminating the D-layer (though not yet the ground). The low-band reflectivity reduction sharply overshoots the mean low-band daytime value; the high band shows no such dawn overshoot. 
4. The sunset transition is not simultaneous between the bands. It begins as the sun sets past $Z \sim 70-75$ deg for the high band but as the sun sets past $Z \sim 85-90$ deg for the low band.

5. The zenith dependence of the reflectivity is far more cusp-like than is the relatively smooth zenith dependence of the ionospheric-reflection height $H_{i}$ seen in $\mathbf{J} 7$ (see in particular Fig. 5 in J7).

Further removal of systematic variations is now implemented:

- We subtract the grazing-incidence logarithmic medians (Fig. 6) from the logarithmic reflectivities of all the data in the range $-140<Z<+140 \mathrm{deg}$. The medians are subtracted bin-by-bin in zenith. Due to their rather sharp and asymmetric features, the bin medians of Fig. 6 are not fitted to a function of zenith, but are used directly, in tabular form. This step leads to "zenith-regressed" logarithmic reflectivities of all the data in the range $-140<Z<+140$ deg.

- Next, we regress the the trends versus $\cos \left(\theta_{i}\right)$, for both daytime and nighttime, and for both low-band and highband.

Having detrended the energy reflectivity with respect to both zenith and angle-of-incidence, we now isolate the effect of $T$-to- $R$ (see Fig. 1) propagation azimuth. Pure-nighttime $(|Z|>110 \mathrm{deg})$ data, detrended with respect to both zenith and angle-of-incidence, are shown in Fig. 7, for (a) low- and (b) high-band data. Each band's azimuth variation is fitted to a two-harmonic function $F(a)$, shown by a grey curve:

$F(a)=A_{1} \cos \left(2 \pi a+\phi_{1}\right)+A_{2} \cos \left(4 \pi a+\phi_{2}\right)$

where $a$ is the azimuth in radians, $A_{1,2}$ are amplitudes, and $\phi_{1,2}$ are azimuth offsets. Figure 7a shows that the low-band residual variance (after detrending with respect to both zenith and angle-of-incidence) is about $50 \%$ controlled by azimuth. The peak reflectivity occurs near $+90 \mathrm{deg}$, which is to say, for propagation from West toward East. The minimum reflectivity is for propagation from East toward West. By contrast, the nighttime high-band data's variations (Fig. 7b) are less controlled by azimuth than are the low-band's.

Figure 8 is similar to Fig. 7 but for pure-daytime conditions $(|Z|<70 \mathrm{deg})$. Again, the low-band variability is about $50 \%$ controlled by azimuth, while azimuth controls less of the high-band variability. We are unaware of previous reports of daytime VLF/LF propagation sensitivity to azimuth.

\section{Frequency-resolved statistical results}

At the risk of less-robust statistics, we can examine the results using frequency as an independent sorting variable. Although we will show all twelve Fourier frequencies in

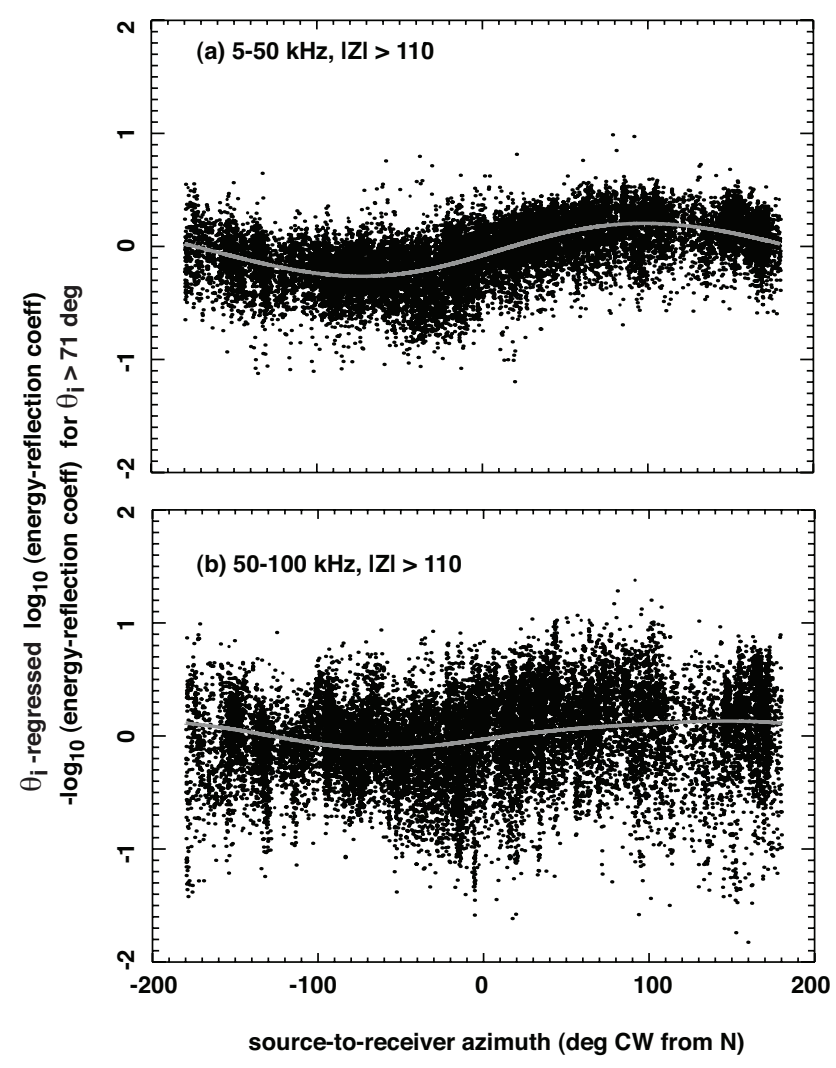

Fig. 7. Propagation-azimuth dependence of zenith- and rangeregressed data for pure-nighttime values of solar zenith angle. Sinusoidal (fundamental + second harmonic) fit is shown as smooth curve.

the range $0<f<100 \mathrm{kHz}$, there are in fact only three independent frequency parameters, due to the initial quadratic fit applied to each event's reflection spectrum (see Fig. 4 and Sect. 2.3). In order to avoid the unstable time periods (see Fig. 6) around each solar terminator, we will restrict the frequency-sorted results analysis to either pure-nighttime $(|Z|>110 \mathrm{deg})$ or pure-daytime $(|Z|<70 \mathrm{deg})$ data. Interior to each of these $|Z|$ ranges, we will ignore variability due to $|Z|$. Figure 6 suggests that this is a viable approximation.

Figure 9 shows average $\log _{10}$ (reflectivity) versus frequency for four different ranges of ionospheric angle of incidence, from (a) steepest-incidence to (d) most grazing incidence. Dashed curves show the pure-nighttime data, solid curves the pure-daytime data. The discrete symbols are the Fourier frequencies. Each point is the average over all events in the $Z$ and $\theta_{i}$ range box. These data have not been detrended against systematic variations with either $Z$ or azimuth. We note:

1. Pure-nighttime reflectivity is practically flat versus frequency, whereas pure-daytime reflectivity decreases (by up to two orders of magnitude, at steepest incidence in Fig. 9a) across the spectrum. 


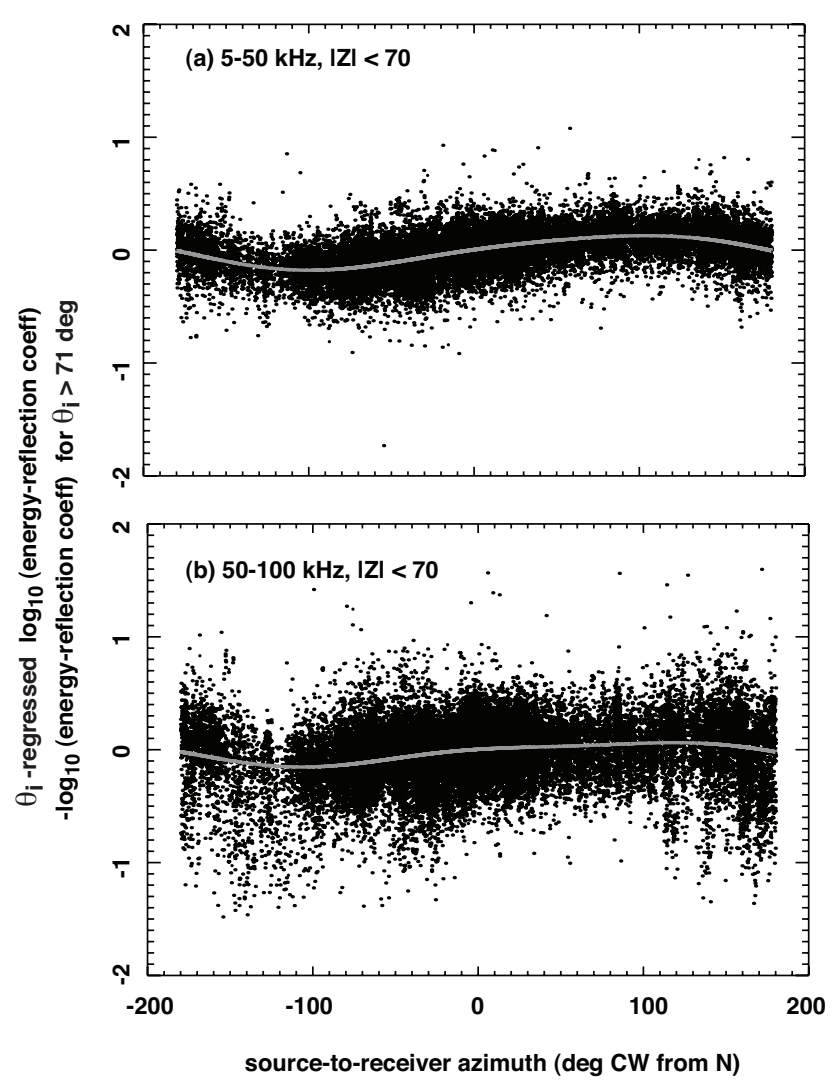

Fig. 8. Propagation-azimuth dependence of zenith- and rangeregressed data for pure-daytime values of solar zenith angle. Sinusoidal (fundamental + second harmonic) fit is shown as smooth curve.

2. Daytime reflectivity roll-off with frequency is strongest for steep incidence (Fig. 9a) and weakest for grazing incidence (Fig. 9d).

\section{Discussion}

We now discuss points of contact between our data and prior results by other investigators.

A surprising feature of the zenith-angle dependence of low-band reflectivity in Fig. 6 is the overshoot downward at the sunrise transition. There is also a small sunset overshoot, though weaker. A comparable sunrise-overshoot effect is seen in some of the data from VLF-amplitude long-range beacon recordings (see Figs. 2-3, left column, in McRae and Thomson, 2000). This is noteworthy, because the beacon data are from long (up to $10^{4} \mathrm{~km}$ ) trans-oceanic paths, whereas our paths are only a few-hundred km. Earlier works based on data from the 1950s are not particularly instructive on this effect (but see Thomas and Harrison, 1970).

Another noteworthy feature of the terminator transitions in Fig. 6 is the large asymmetry in timing between sunrise and sunset: The terminator occurs simultaneously on low- band and high-band reflectivity at sunrise, but not at sunset, where the low-band recovery to nighttime levels is delayed with respect to the high-band. We are unaware of previous observations of this effect. Early bistatic VLF-propagation measurements were based on a recording of the radio signal from single-frequency. The more recent broadband-VLF approach of Cummer has been used exclusively during purenighttime conditions and has not been applied to terminator behavior. Recently a colleague (J. Zinn, private communication) has pointed out that our observed dawn transient at low band is probably due to prompt photo-detachment of electrons from nocturnal negative ions. The first rays of dawn cause a fast supply of free electrons in this manner. This effect has been predicted by a model of D-region photochemistry, but the effect eluded confirmation by the Arecibo incoherent-scatter radar due to that methods lack of sensitivity at D-region heights (Ganguly and Zinn, 1998).

Our empirical retrieval of the energy-reflectivity's azimuth sensitivity, during both nighttime (Fig. 7) and daytime (Fig. 8), shows a peak in the reflectivity for "west-to-east" and a minimum for "east-to-west" propagation. The lowband data reflectivity variation versus azimuth is roughly sinusoidal. The high-band variation is noisier due to the lower ionospheric-echo SNR at higher frequencies and will not be discussed further.

The prior published information about observational evidence for azimuth effects on VLF reflectivity appears to condense down to a handful of reports. Two of these reports are from the United States Naval Electronics Laboratory Center or NELC (subsequently named Naval Ocean Systems Center or NOSC) and deal with the same dataset, gathered in 1969. The earlier of these reports (Bickel et al., 1970) compared signal-propagation paths from Hawaii roughly east-to-west to Wake Island, and from Hawaii roughly west-to-east to southern California. The signal was the $23.4-\mathrm{kHz}$ transmission from the NPM beacon in Hawaii. Airborne amplitude recordings, conducted continuously along flights over portions of the propagation baselines, observed that there was more attenuation on the east-to-west than on the west-to-east path (see Figs. 7-8 and discussion thereof, in Bickel et al., 1970). On the other hand, the amplitudes on the east-to-west path were highly variable from day to day. The second of the NELC/NOSC reports (Pappert and Hitney, 1988) addresses this observed anisotropy and irreproducibility (in the eastto-west measurements) with detailed model calculations, but uses the same data as that of Bickel et al.

The other prior independent report of reflection anisotropy (Grandt, 1992) is based on VLF sferic recordings. Rather than in amplitude, the reported anisotropy is in the signal's group delay dispersion ("gdd"). The gdd concept has been clearly reviewed elsewhere (Figure Eq. 3.49, and discussion thereof, in vol. 2, chapter 3, "Longwave Sferics Propagation within the Atmospheric Waveguide", in Volland, 1995). At any rate, the evidence of the sferic recordings (see Fig. 2 in Grandt, 1992) appears to suggest that there may be 
(a) $46<\theta_{\mathbf{i}}<58 \mathrm{deg}$

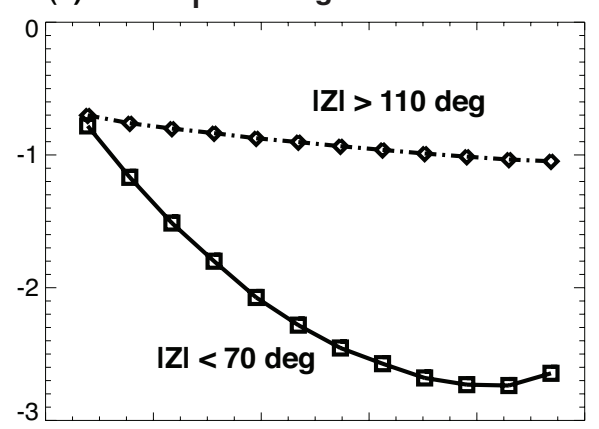

(c) $62<\theta_{\mathrm{i}}<67 \mathrm{deg}$

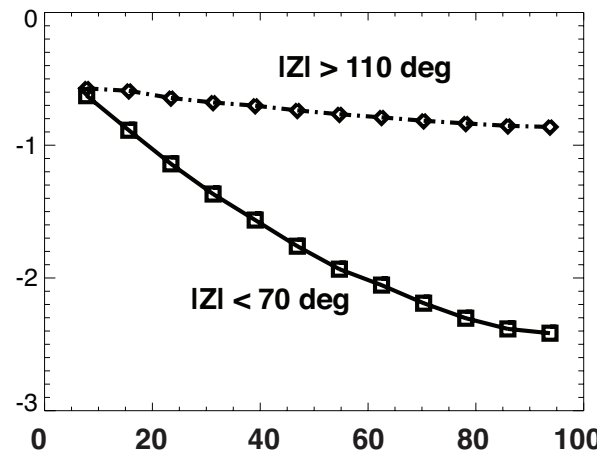

(b) $58<\theta_{\mathrm{i}}<62 \mathrm{deg}$

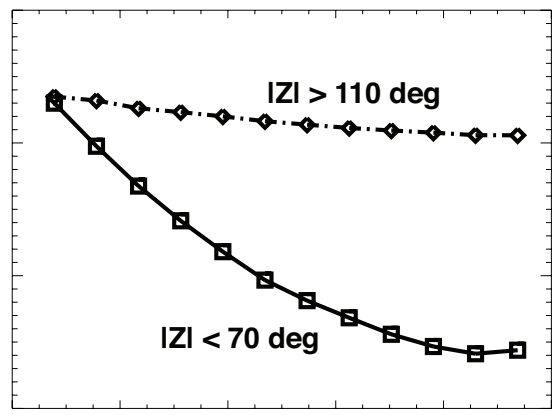

(d) $67<\theta_{\mathbf{i}}<80 \mathrm{deg}$

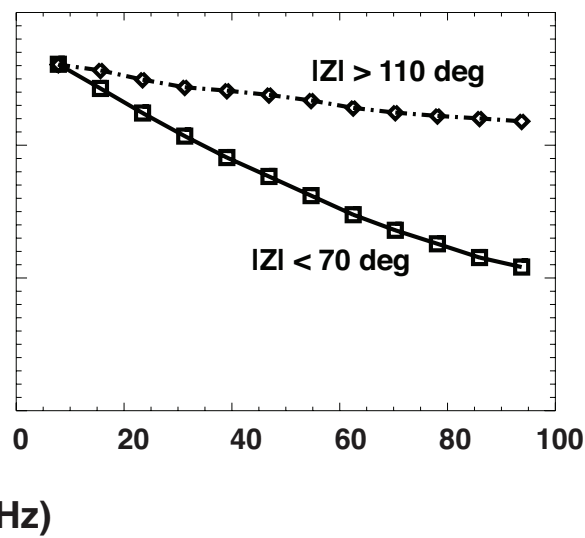

Fig. 9. Average $\log _{10}$ (reflectivity) versus frequency for four different ranges of ionospheric angle of incidence, from (a) steepest-incidence to (d) most grazing incidence. Dashed curves show the pure-nighttime data, solid curves the pure-daytime data. The discrete symbols are the Fourier frequencies. Each point is the average over all events in the $Z$ and $\theta_{i}$ range box. These data have not been detrended against systematic variations with either $Z$ or azimuth. The smooth variation versus frequency is primarily due to our reduction of the frequency information to just a quadratic fit (see Fig. 4 above) in analyzing each event's reflection. This smooth variation may mask details of the frequency response that cannot be captured by a quadratic fit.

a group-delay-dispersion difference between two azimuths, one near $90 \mathrm{deg}$ and the other near $270 \mathrm{deg}$. Our reading of Grandt's report is that its Fig. 2 fails to separate the effects of distance and azimuth. Moreover, there is no direct data on reflection amplitude.

In summary, then, the only journal-published, hard observations on amplitude anisotropy appear to be that of NELC/NOSC's flights along two discrete azimuths. We infer, then, that the results in Figs. 7 (for nighttime) and 8 (for daytime) are quite new, at least in their making obvious the azimuthal variation of the single-hop ionospheric reflectivity. Moreover, the results in Fig. 8 are the first report of daytime VLF azimuth sensitivity, to the best that we can ascertain. We note, however, that NELC/NOSC has produced a wealth of reports on results of airplane-borne beacon recordings, which although not published in journals, were available as microphiches (as recently as 1995) from the United States National Technical Information Service. These reports are said to contain scattered information on the subject of magnetic anisotropy.
The second NELC/NOSC article (Pappert and Hitney, 1988) hypothesizes a mechanism for the irreproducibility of the east-to-west propagation amplitude relative to the west-to-east propagation: the east-to-west-propagating mode shape extends higher in altitude than does the west-to-eastpropagating mode shape. This increases the susceptibility of east-to-west propagation to sporadic-E irregularities in the heights above $90 \mathrm{~km}$. Sporadic-E is notoriously irregular in time and position, so it might introduce irreproducibility into VLF waveguide propagation modes that extend high enough to sample the sporadic-E. Examining our nighttime data (Fig. 7) however, we see no obvious tendency for greater reflectivity scatter at east-to-west azimuth compared to westto-east azimuth.

Acknowledgements. This work would not have been possible without those who built and operated the early LASA system, including Robert Massey, Kyle Wiens, David A. Smith, Ken Eack, Jeremiah Harlin, and Matthew Heavner.

We have benefited from useful discussions with Neil R. Thomson and Steven A. Cummer. 
The LASA array has been operated under the auspices of the United States Department of Energy.

Topical Editor M. Pinnock thanks K. Eack and another anonymous referee for their help in evaluating this paper.

\section{References}

Bickel, J. E., Ferguson, J. A., and Stanley, G. V.: Experimental observation of magnetic field effects on VLF propagation at night, Radio Sci., 5, 19-25, 1970.

Bortnik, J., Inan, U. S., and Bell, T. F.: Temporal signatures of radiation belt electron precipitation induced by lightning-generated MR whistler waves: 1. Methodology, J. Geophys. Res., 111, A02204, doi:10.1029/2005JA011182, 2006a.

Bortnik, J., Inan, U. S., and Bell, T. F.: Temporal signatures of radiation belt electron precipitation induced by lightning-generated MR whistler waves: 2. Global signatures, J. Geophys. Res., 111, A02205, doi:10.1029/2005JA011398, 2006b.

Bracewell, R. N., Budden, K. G., Ratcliffe, J. A., Straker, T. W., and Weekes, K.: The ionospheric propagation of low- and very-lowfrequency radio waves over distances less than $1000 \mathrm{~km}$, Proc. Instn. Elect. Engrs., 98, 221-236, 1951.

Cheng, Z. and Cummer, S. A.: Broadband VLF measurements of lightning-induced ionospheric perturbations, Geophys. Res. Lett., 32, L08804, doi:10.1029/2004GL022187, 2005.

Cheng, Z., Cummer, S. A., Baker, D. N., and Kanekal, S. G.: Nighttime D region electron density profiles and variabilities inferred from broadband measurements using VLF radio emissions from lightning, J. Geophys. Res., 111, A05302, doi:10.1029/2005JA011308, 2006.

Cho, M. and Rycroft, M. J.: Computer simulation of the electric field structure and optical emission from cloud-top to the ionosphere, J. Atmos. Sol.-Terr. Phys., 60, 871-888, 1998.

Cummer, S. A.: Lightning and ionospheric remote sensing using VLF/ELF radio atmospherics, Department of Electrical Engineering, Stanford University, 152, 1997.

Cummer, S. A., Inan, U. S., and Bell, T. F.: Ionospheric D region remote sensing using VLF radio atmospherics, Radio Sci., 33, 1781-1792, 1998.

Deeks, D. G.: D-region electron distributions in middle latitudes deduced from the reflexion of long radio waves, Proc. Roy. Soc. Lon. A, 291, 413-437, 1966.

Ganguly, S. and Zinn, J.: Time-variations of D region electron densities, and comparison with model computations, Studia Geoph. et Geod., 42, 500-510, 1998.

Grandt, C.: Thunderstorm monitoring in South Africa and Europe by means of Very Low Frequency sferics, J. Geophys. Res., 97, 18 215-18 226, 1992.

Inan, U. S., Sampson, W. A., and Taranenko, Y. N.: Space-time structure of optical flashes and ionization changes produced by lightning-EMP, Geophys. Res. Lett., 23, 133-136, 1996.

Inan, U. S., Pasko, V. P., and Bell, T. F.: Sustained heating of the ionosphere above thunderstorms as evidenced in "early/fast" VLF events, Geophys. Res. Lett., 23, 1067-1070, 1996.

Jacobson, A. R. and Heavner, M. J.: Comparison of Narrow Bipolar Events with ordinary lightning as proxies for severe convection, Mon. Weather Rev., 133, 1144-1154, 2005.

Jacobson, A. R., Boeck, W., and Jeffery, C.: Comparison of Narrow Bipolar Events with ordinary lightning as proxies for the microwave-radiometry ice-scattering signature, Mon. Weather Rev., 135, 1354-1363, 2007.

Jacobson, A. R., Holzworth, R., Lay, E., Heavner, M., and Smith, D. A.: Low-frequency ionospheric sounding with Narrow Bipolar Event lightning radio emissions: Regular variabilities and solarX-ray responses, Ann. Geophys., 25, 2175-2184, 2007, http://www.ann-geophys.net/25/2175/2007/.

Johler, J. R. and Harper Jr., J. D.: Reflection and transmission of radio waves at a continuously stratified plasma with arbitrary magnetic induction, J. Res. Nat. Bur. Stand.-D., 66, 81-99, 1962.

Le Vine, D. M.: Sources of the strongest rf radiation from lightning, J. Geophys. Res., 85, 4091-4095, 1980.

Lev-Tov, S. J., Inan, U. S., and Bell, T. F.: Altutude profiles of localized D region density disturbances produced in lightninginduced electron precipitation events, J. Geophys. Res., 100, $21375-21383,1995$.

McRae, W. M. and Thomson, N. R.: VLF phase and amplitude: daytime ionospheric parameters, J. Atmos. Sol.-Terr. Phys., 62, 609-618, 2000.

Pappert, R. A. and Ferguson, J. A.: VLF/LF mode conversion model calculations for air to air transmissions in the Earth-ionosphere waveguide, Radio Sci., 21, 551-558, 1986.

Pappert, R. A. and Hitney, L. R.: Empirical modeling of nighttime easterly and westerly VLF propagation in the Earth-ionosphere waveguide, Radio Sci., 23, 599-611, 1988.

Piggott, W. R., Pitteway, M. L. V., and Thrane, E. V.: The numerical calculation of wave-fields, reflexion coefficients and polarizations for long radio waves in the lower ionosphere. II, Phil. Trans. Roy. Soc. Lon., 257, 243-271, 1965.

Pitteway, M. L. V.: The numerical calculation of wave-fields, reflexion coefficients and polarizations for long radio waves in the lower ionosphere. I, Phil. Trans. Roy. Soc. Lon., 257, 219-241, 1965.

Rodger, C. J., Clilverd, M. A., Thomson, N. R., Nunn, D., and Lichtenberger, J.: Lightning driven inner radiation belt energy deposition into the atmosphere: regional and global estimates, Ann. Geophys., 23, 3419-3430, 2005, http://www.ann-geophys.net/23/3419/2005/.

Smith, D. A., Heavner, M. J., Jacobson, A. R., Shao, X. M., Massey, R. S., Sheldon, R. J., and Wiens, K. C.: A method for determining intracloud lightning and ionospheric heights from VLF/LF electric field records, Radio Sci., 39, doi:10.1029/2002RS002790, 2004.

Smith, D. A., Eack, K. B., Harlin, J., Heavner, M. J., Jacobson, A. R., Massey, R. S., Shao, X. M., and Wiens, K. C.: The Los Alamos Sferic Array: A research tool for lightning investigations, J. Geophys. Res., 107, 4183, doi:10.1029/2001JD000502, 2002.

Smith, D. A., Shao, X. M., Holden, D. N., Rhodes, C. T., Brook, M., Krehbiel, P. R., Stanley, M., Rison, W., and Thomas, R. J.: A distinct class of isolated intracloud lightning discharges and their associated radio emissions, J. Geophys. Res., 104, 4189-4212, 1999.

Suszcynsky, D. M. and Heavner, M. J.: Narrow Bipolar Events as indicators of thunderstorm convective strength, Geophys. Res. Lett., 30, 1879, doi:10.1029/2003GL017834, 2003.

Thomas, L. and Harrison, M. D.: The electron density distributions in the D-region during the night and pre-sunrise period, J. Atmos. Terr. Phys., 32, 1-14, 1970. 
Thomson, N. R.: Experimental daytime VLF ionospheric parameters, J. Atmos. Terr. Phys., 55, 173-184, 1993.

Thomson, N. R. and Clilverd, M. A.: Solar flare induced ionospheric D-region enhancements from VLF amplitude observations, J. Atmos. Sol.-Terr. Phys., 63, 1729-1737, 2001.

Thomson, N. R. and Rodger, C. J.: Large solar flares and their ionospheric D region enhancements, J. Geophys. Res., 110, A06306, doi:10.1029/2005JA011008, 2005.

Thomson, N. R., Rodger, C. J., and Dowden, R. L.: Ionosphere gives size of greatest solar flare, Geophys. Res. Lett., 31, L06803, doi:10.1029/2003GL017345, 2004.

Thomson, N. R., Clilverd, M. A., and McRae, W. M.: Nighttime ionospheric $\mathrm{D}$ region parameters from VLF phase and amplitude, J. Geophys. Res., 112, A07304, doi:10.1029/2007JA012271, 2007.
Volland, H. (Ed.): Handbook of atmospheric electrodynamics, Vol. 2CRC Press, 1995.

Wait, J. R. and Spies, K. P.: NBS Technical Note 300: Characteristics of the Earth-ionosphere waveguide for VLF radio waves, edited by: U. S. D. o. C. National Bureau of Standards (now National Institute of Standards and Technology, NBS, 1964.

Willett, J. C., Bailey, J. C., and Krider, E. P.: A class of unusual lightning electric field waveforms with very strong highfrequency radiation, J. Geophys. Res., 94, 16 255-16 267, 1989. 